\title{
Lymphocyte to Monocyte Ratio and monocyte subtypes are predictors of severity and outcome in acute ischemic stroke
}

Marta Oses ${ }^{* 1,4}$, Alicia García-Culebras*4, MA García- Torres ${ }^{1}$, Violeta Durán-Laforet ${ }^{4}$, Carolina Peña-Martínez ${ }^{4}$, Inmaculada Navas ${ }^{1}$, Cristina Serrano² Fernando Ostos ${ }^{3}$, Jaime Díaz-Guzmán ${ }^{3}$, Pilar Llamas², MA Moro ${ }^{4}$ Ignacio Lizasoaín ${ }^{4}$

1.Stroke Unit. Neurology Department. Hospital Universitario Fundación Jiménez Díaz, Madrid, Spain

2. Haematology Department. Hospital Universitario Fundación Jiménez Díaz, Madrid, Spain

3.Stroke Unit. Neurology Department. Hospital Universitario 12 de Octubre, Madrid, Spain

4. Neurovascular Research Unit, Department of Pharmacology and Toxicology and Neurochemistry Research Institute, Universidad Universidad Complutense de Madrid, Spain and i+12 Health Research Institute, Madrid, Spain.

\section{INTRODUCTION}

- Monocytes are heterogeneous cells that play a key role in the pathophysiology of acute ischemic stroke.

- Monocytes can adopt proinflammatory or anti-inflammatory phenotypes according to their expression of lipopolysaccharide receptor (CD14) and Fcg receptor III (CD16)

- Low lymphocyte to monocyte ratio (LMR) has recently been associated with stroke severity and poor long-term outcome.

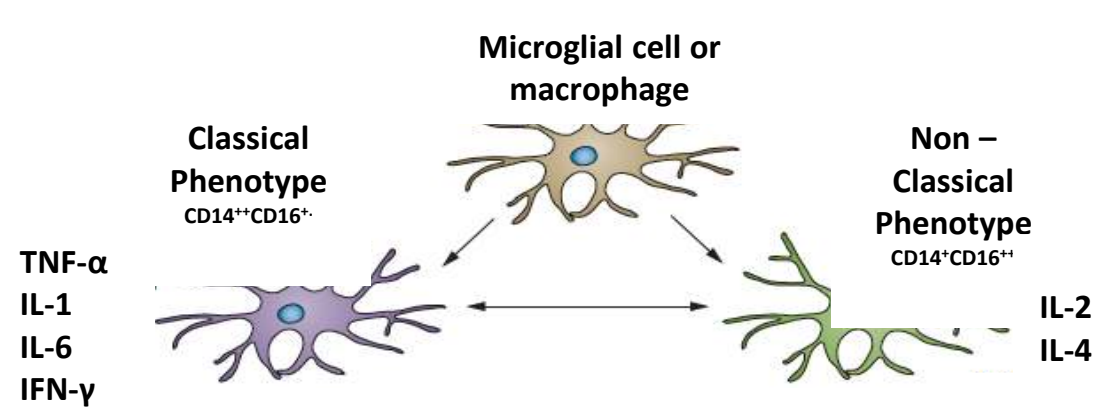

\section{OBJECTIVES \& METHODS}

We assessed whether LMR, the different monocytes subsets and its cytokines can predict the clinical course and outcome of patients who suffer an acute ischemic stroke (AIS) or transient ischemic attack (TIA)

- Prospective case - control study

- Group of AIS and TIA patients and control group from two stroke units.

- Three blood and plasma samples were collected at acute stage

- Flow cytometry study done at 48 hours

- Quantitative analysis of different cytokines present in the plasma using a customised Cytometric Bead array were performed: IFN-y, TNF- $\alpha$, IL-6, IL-4, IL-2

- Neurological evaluation of severity was assessed using NIHSS scale and functional outcome using the modified Rankin Scale (mRS)

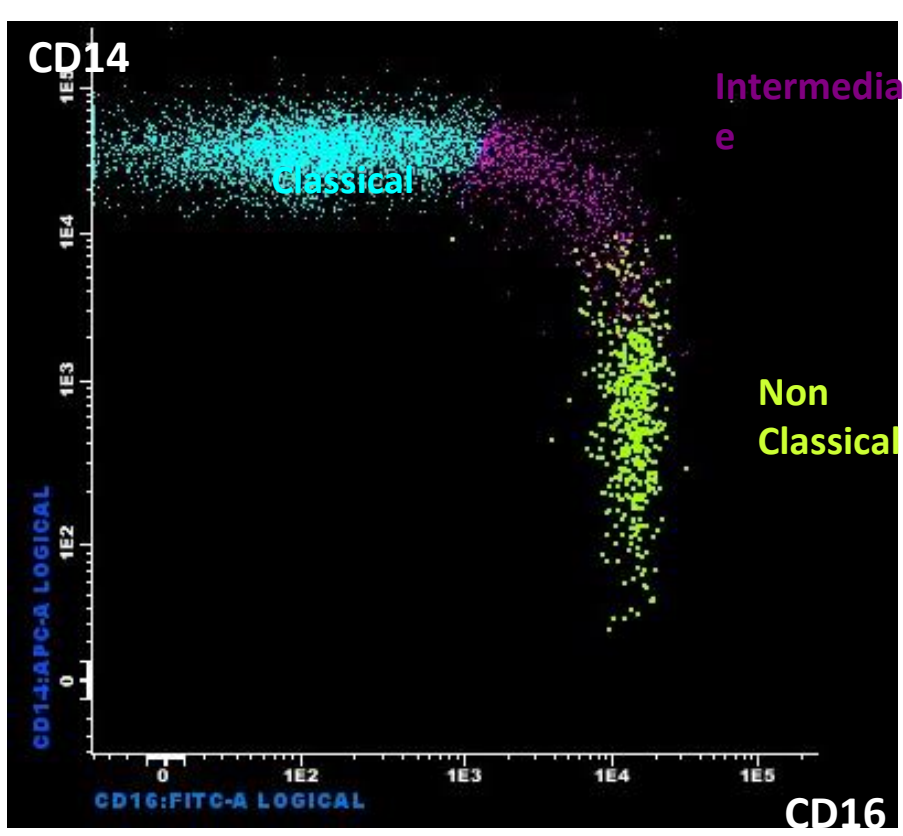

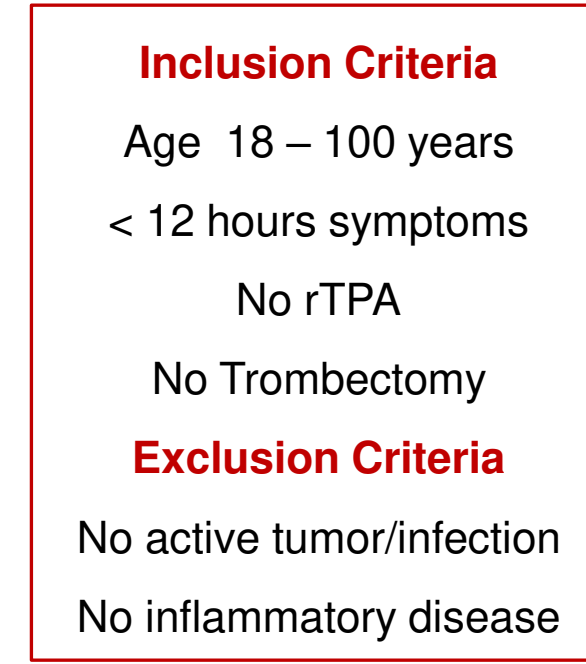

Image 1. Monocytes populations in Flow Cytometry

\begin{tabular}{|lll|}
\hline Subsets & Surface Markers & Functions \\
Classical & $\mathrm{CD} 14^{++} \mathrm{CD} 16^{-}$ & Phagocytosis \\
Intermediate & $\mathrm{CD} 14^{++} \mathrm{CD} 16^{+}$ & Proinflammatory \\
Non-classical & $\mathrm{CD} 14^{+} \mathrm{CD} 16^{++}$ & Patrolling \\
\hline
\end{tabular}

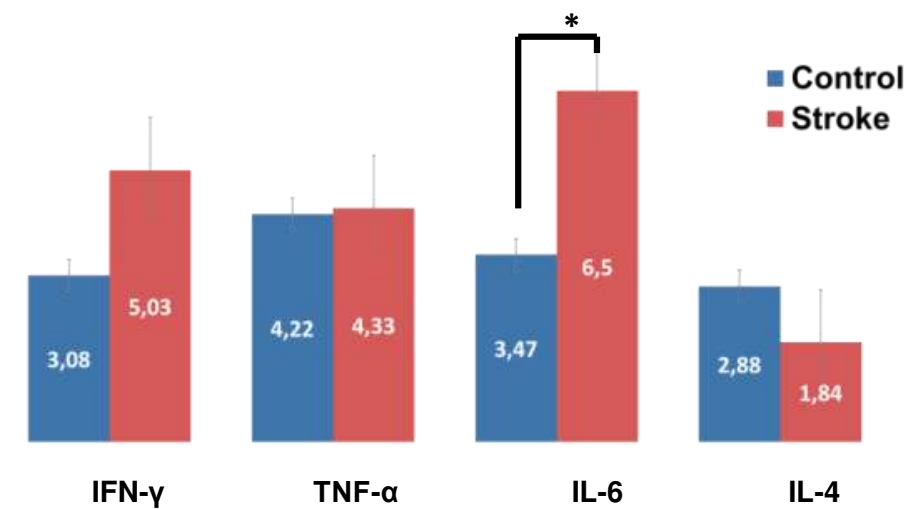

Image 4. Cytokines at acute stage

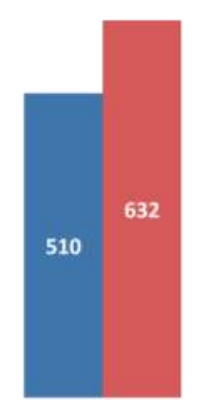

Total
Monocytes
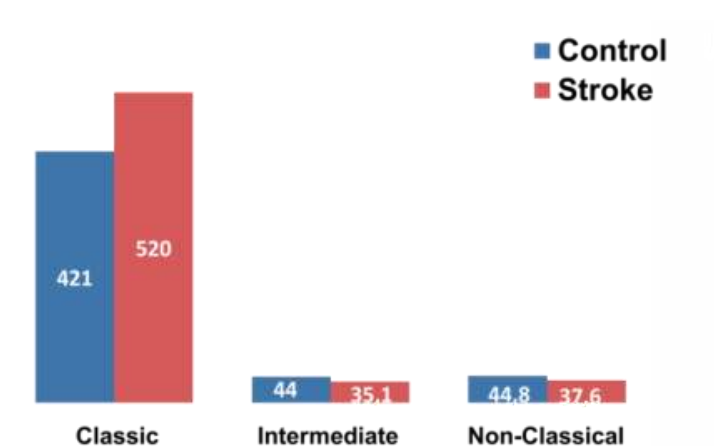

Image 2. Monocytes populations in study and control group

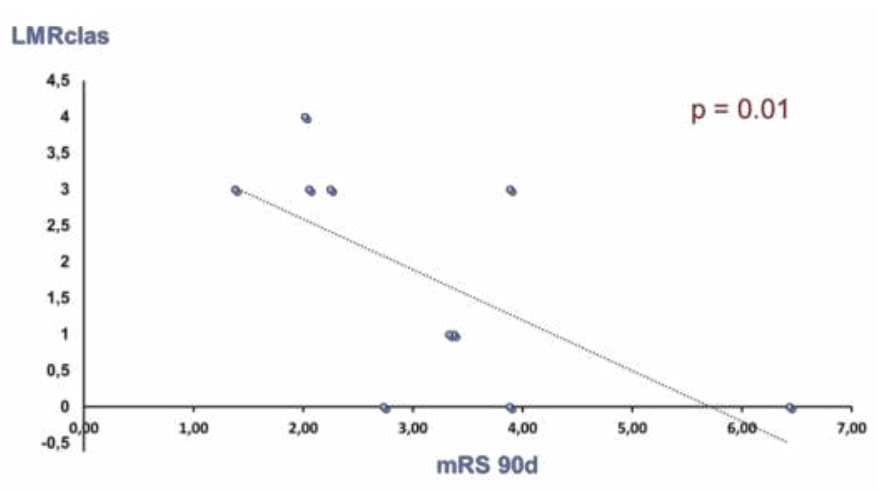

Image 3. LMR Classic is associated with functional outcome (mRS) at day 90

\section{RESULTS}

11 patients, 5 controls

- Median age 79 years

- $72 \%$ High blood pressure

- Older patients had higher NIHSS and higher mRS

- Lower LMR at 48 hours is associated with higher mRS at 90 days $(p=0.01)$ $m R S$ at 90 days $(p<0.01)$. This association was not seen with intermediate monocytes (IM; CD14+CD16+) and non-classical monocytes (NCM; CD14CD16++)

The results show a trend to higher total monocyte count and classical monocyte

Total monocyte count is associated with higher NIHSS at 24 hours and Classical monocytes with higher NIHSS at $24 \mathrm{~h}$ and higher mRS at 90 days, with a trend toward significance $(p=0.07)$. This association was not seen with IM and NCM

Baseline levels of IL-6 were higher in study group and was associated with higher basal NIHSS $(p<0.02)$ 